\title{
ACOUSTO-OPTICS IN SCATTERING MEDIA: AN ANALYTICAL MODEL FOR ANISOTROPIC SCATTERING
}

\author{
Sava Sakadžić and Lihong V. Wang \\ Optical Imaging Laboratory, Biomedical Engineering Program, \\ Texas A\&M University, 3120 TAMU, College Station, Texas 77843-3120, \\ http://oilab.tamu.edu
}

\begin{abstract}
We have calculated analytically the temporal autocorrelation function of the electric field component of multiply scattered coherent light transmitted through an anisotropically scattering mediuma irradiated with a plane ultrasonic wave. The accuracy of the analytical solution is verified with an independent Monte Carlo simulation. The analytical model shows that an approximate similarity relation exists.
\end{abstract}

Keywords-Ultrasonic modulation, multiply scatterd light, autocorrelation function, Monte Carlo.

\section{INTRODUCTION}

Recently ultrasound-modulated optical tomography has been established as a new and growing area of research. Potential applications exist in the imaging of scattering media, especially biological tissues. This technique combines ultrasonic resolution and optical contrast based on the differences in optical properties among different types of tissues. Several groups have conducted intensive research in the past few years $[1-10]$ in an attempt to explain the mechanism of ultrasonic tagging of light and to develop practical systems based on this new imaging modality.

In our simple model we will consider two basic mechanisms that are responsible for variation in the optical phase of multiply scattered light: the ultrasound-induced collective displacements of scatterers and the ultrasoundinduced variation of the index of refraction [8], [9], [10].

In this paper we extend the solution for the temporal autocorrelation function of the electrical field component for isotropic scattering media obtained in [8], incorporating into the model a general scattering phase function. Section II describes the derivation of the autocorrelation function along paths of length $s$. In Section III, the obtained expression is incorporated into the solution for the total electric field autocorrelation function transmitted through a scattering slab. We examine the accuracy of our analytical solution with an independent Monte Carlo simulation. Finally, a brief summary of our conclusions is presented.

\section{AUtOCORRELATION FOR A Single PATHLENGTH}

Consider the propagation of coherent light through a homogeneous scattering medium irradiated by a plane ultrasonic wave. In a weak scattering approximation the temporal autocorrelation function of the electric field component of the scattered light at the point detector position can be written as follows [7], [8], [10]:

$G_{1}(\tau)=\int_{0}^{\infty} p(s)\left\langle E_{s}(t) E_{s}^{*}(t+\tau)\right\rangle_{U}\left\langle E_{s}(t) E_{s}^{*}(t+\tau)\right\rangle_{B} d s$ where $p(s)$ is the probability density function of path length $s$. In (1) we assume that contributions from Brownian motion $(B)$ and ultrasound $(U)$ are independent and that we can separate them.

Following the derivations in [8], [10], we can write:

$$
\left\langle E_{s}(t) E_{s}^{*}(t+\tau)\right\rangle_{U}=\exp (-F(\tau) / 2),
$$

$$
F(\tau)=\exp \left\langle\left[\sum_{j=1}^{N} \Delta \phi_{n, j}(t, \tau)+\sum_{j=1}^{N-1} \Delta \phi_{d, j}(t, \tau)\right]^{2}\right\rangle
$$

In (3), $\Delta \phi_{n, j}(t, \tau)$ and $\Delta \phi_{d, j}(t, \tau)$ are respectively the differences between phase variations induced by the modulated index of refraction along the $j$ th free path, and by the modulated displacement of the $j$ th scatterer following the $j$ th free path [8]. Summation is going over all $N$ free paths (represented by vectors $\mathbf{l}_{j}=l_{j} \hat{\mathbf{e}}_{j}$ ) and $N-1$ scattering events (at positions $\mathbf{r}_{j}$ ) along the photon path. Averaging is over time and over all the photon paths of length $s$.

The probability density function $p\left(\mathbf{l}_{1}, \ldots, \mathbf{l}_{N}\right)$ of a particular photon path in diffusion regime can be approximated as follows

$$
p\left(\mathbf{l}_{1}, \ldots, \mathbf{l}_{N}\right)=p_{s}\left(\hat{e}_{1}\right) \prod_{j=1}^{n} p\left(l_{j}\right) \prod_{j=1}^{N-1} f\left(\hat{\mathbf{e}}_{j} \cdot \hat{\mathbf{e}}_{j+1}\right),
$$

where $p\left(l_{j}\right)=l^{-1} \exp \left(-l_{j} / l\right)$ is the probability density of a photon free path, $l$ is the photon mean free path, $f()$ is the scattering phase function, and $p_{s}\left(\hat{\mathbf{e}}_{1}\right)$ is the probability density function of the starting photon direction $\hat{\mathbf{e}}_{1}$.

After going through some algebra, equation (3) becomes

$$
F(\tau) \simeq s\left(2 n_{0} k_{0} A\right)^{2} \sin ^{2}\left(\omega_{a} \tau / 2\right)\left(\delta_{n}+\delta_{d}\right) .
$$

In (5), $n_{0}$ is the index of refraction, $k_{0}$ is the optical wave vector, $A$ and $\omega_{a}$ are the ultrasonic amplitude, and angular frequency. Terms $\delta_{n}=\eta^{2} k_{a}^{2} l \Re$ and $\delta_{d}=(1-g) /(3 l)$ represents respectively the index of refraction and the scatterer displacement influence on the autocorrelation function. $k_{a}$ is ultrasonic wave vector, $g$ is the scattering anisotropy, and $\eta$ is equal to $(\partial \eta / \partial p) \rho v_{a}^{2}\left(v_{a}\right.$ is the acoustic velocity, $\rho$ is medium density, and $\partial \eta / \partial p$ is the adiabatic piezo-optical coefficient). $\Re$ represents the real value of the $(0,0)$ element of the matrix $\hat{J}(\hat{I}-\hat{J})^{-1}$ :

$$
J_{m, n}=M \int_{-1}^{1} T(x) P_{m}(x) P_{n}(x) d x
$$

In (6), $T(x)=1 /\left(1-i k_{a} l\right), M^{2}=g_{m} g_{n}(2 m+1)(2 n+1) / 4$, $P_{j}(x)$ is Legendre polynomial of order $j$, and $g_{j}$ is the $j$ th 


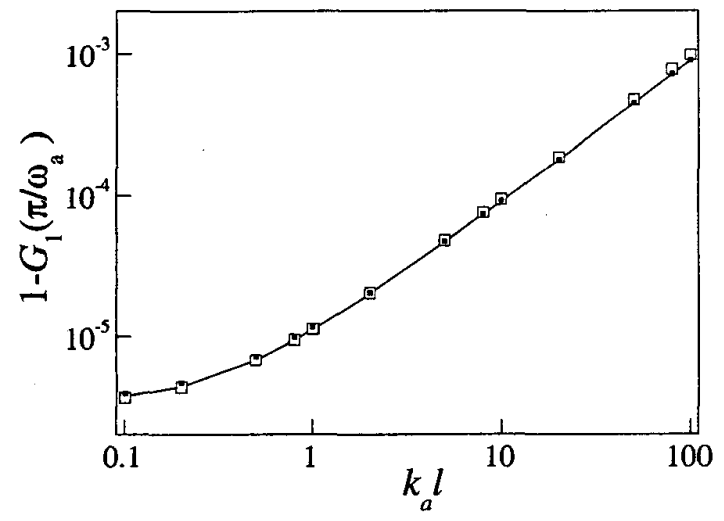

Fig. 1. The $k_{0} l$ dependence of the maximum variation of the time autocorrelation function while $k_{\mathfrak{a}}$ is kept constant. Empty squares indicate the Monte Carlo results. Solid lines indicate the analytical results. Filed squares indicate the analytical results as well but using the similarity relation $\left(L / l=127.35, g=0.9, A=0.1 \AA, \lambda_{0}=500\right.$ $\left.\mathrm{nm}, n_{0}=1.33, f_{a}=1 \mathrm{MHz}, v_{a}=1480 \mathrm{~m} / \mathrm{s}, \eta=0.3211\right)$.

Legendre polynomial expansion coefficient of the scattering phase function.

\section{AUTOCORRELATION FOR A SLAB}

Slab geometry has been considered previously for various particular problems [7-13]. We will solve (1) for anisotropically scattering and absorbing media based on the expression for $F(\tau)$ obtained in the previous section.

One side of the slab is irradiated by a plane electromagnetic wave, and a point detector measures the temporal autocorrelation function of the electric field on the other side of the slab. We can solve the integration in (1) over $s$ for the temporal autocorrelation function incorporating the influence of Brownian motion of scatterers [7], [10], [12] and expression for $F(\tau)$ obtained in Sec. II. The photon path length probability density function $p(s)$ is found by solving the diffusion equation for such geometry [8], [11], [13]. The result of integration is:

$$
G_{1}(\tau)=\left[\operatorname{sh}\left(L_{0} K\right) / \operatorname{sh}\left(z_{0} K\right)\right]\left[\operatorname{sh}\left(z_{0} W\right) / \operatorname{sh}\left(L_{0} W\right)\right]:
$$

In $(7), W^{2}=\left(S_{U}+S_{B}+\mu_{a}\right) D^{-1}, S_{B}=2 \tau /\left(\tau_{0} l^{*}\right)$ is the term due to Brownian motion ( $\tau_{0}$ is the single-particle relaxation time), and $S_{U}$ is the term due to the ultrasonic influence $S_{U}=F(\tau) /(2 s) . K^{2}=\mu_{a} D^{-1}$, where $D=l^{*} / 3$ is diffusion constant, $l^{*}=l /(1-g)$ is the isotropic scattering mean free path, and $\mu_{a}$ is absorption coefficient. The distance between the extrapolated boundary and the corresponding real boundary of the slab is $l^{*} \gamma(\gamma=0.7104)$. The converted isotropic source is one isotropic scattering mean free path into the slab. Therefore, $L_{0}=L+2 l^{*} \gamma(L$ is the slab tickness), and $z_{0}=l^{*}(1+\gamma)$.

To provide an independent numerical approach, we modified the existing public domain Monte Carlo package [9], [14] for the transport of light in scattering media, to sample the autocorrelation function according to (1) and (3).
Fig. 1 shows the $k_{a} l$ dependence of the maximum variation of the time autocorrelation function in a case when scattering anisotropy is 0.9 . The analytical predictions (solid lines in Fig.1) fit the Monte Carlo calculations (empty scatterers) very well. Further, analytical predictions according to similarity relation (filled scatterers) also fit very well both Monte Carlo calculations and analytical predictions. In the latest case, we used analytical solution for the autocorrelation function in a case of isotropic scattering [8], with the reduced photon mean free path $l^{*}=l /(1-g)$.

\section{CONClusion}

In conclusion, we presented an analytical solution for the autocorrelation function of the ultrasound-modulated electric field along a path with $N$ scatterers when scattering is anisotropic. An analytical solution was found for light transmitted through a scattering slab using the plane source and the point detector. Using a Monte Carlo simulation, we verified the accuracy of the analytical solution. We also tested the similarity relation and showed that it is a good approximation in the calculation of the autocorrelation function. Our analytical solution is valid under the following conditions: diffusion regime transport, a small ultrasonic modulation, and the value of $k_{a} l$ is not too small.

\section{REFERENCES}

[1] F.A. Marks, H.W. Tomlinson, and G.W. Brooksby, "A comprehensive approach to brest cancer detection using light: photon localization by ultrasound modulation and tissue characterization by spectral discrimination ", Proc. Soc. Photo-Opt. Instrum. Eng., vol. 1888, pp. 500-510, 1993.

[2] L.-H.V. Wang, S.L. Jacques, and X. Zhao, Opt. Lett., vol. 20, pp. $629-631,1995$.

[3] M. Kempe, M. Larionov, D. Zaslavsky, and A.Z. Genack, J. Opt. Soc. Am A., vol. 14, pp. 1151-1158, 1997.

[4] S. Leveque, A.C. Boccara, M. Lebec, and H. Saint-Jalmes, Opt. Lett., vol. 24, pp. 181-183, 1999.

[5] G. Yao, S.-L. Jiao, and L.-H.V. Wang, Opt. Lett., vol. 25, pp. 734-736, 2000.

[6] A. Lev, Z. Kotler, and B.G. Sfez, Opt. Lett., vol. 25, pp. 378-380, 2000.

7] G. Maret and P.E. Wolf, Z. Phys. B, vol. 65, pp. 409-413, 1987.

[8] L.-H.V. Wang, Phys. Rev. Lett., vol. 87, pp. 043903-(1-4), 2001.

$9]$ L.-H.V. Wang, Opt. Lett., vol. 26, pp. 1191-1193, 2001.

10] W. Leutz and G. Maret, Physica B, vol. 204, pp. 14-19, 1995.

11] M.S. Patterson, B. Chance, and B.C. Willson, Appl. Opt., vol. 28, pp. 2331-2336, 1989.

[12] D.J. Pine, D.A. Weitz, P.M: Chaikin, and E. Herbolzheimer, Phys. Rev. Lett., vol. 60, pp. 1134-1137, 1988.

[13] A. Ishimaru, "Wave Propagation and Scattering in Random Media", Academic, New York, 1978.

[14] L.-H.V. Wang, S.L. Jacques, and L.-Q. Zheng, Comput. Methods Prog. Biomed., vol. 47, pp. 131-146, 1995. 